\title{
The QCD phase diagram: Results and challenges
}

\author{
Jan M. Pawlowski \\ Institut für Theoretische Physik, Universität Heidelberg, Philosophenweg 16, 69120 Heidelberg, Germany \\ ExtreMe Matter Institute EMMI, GSI, Planckstr. 1, 64291 Darmstadt, Germany
}

\begin{abstract}
I review the progress made in recent years with functional methods in our understanding of the QCD phase diagram. In particular I discuss a renormalisation group approach to QCD at finite temperature and chemical potential. Results include the location of the confinement-deconfinement phase transition/cross-over and the chiral phase transition/cross-over lines, their nature as well as their interrelation.
\end{abstract}

Keywords: QCD phase diagram, order parameters, functional renormalisation group

PACS: 05.10.Cc,11.10.Wx,12.38.Aw

\section{Introduction}

Quantum Chromodynamics (QCD) at finite temperature and density is a very active area of research. The equation of state of QCD and the nature of the transition from the hadronic phase with broken chiral symmetry to the chirally symmetric, deconfined quark-gluon plasma phase is of great importance for a better understanding of the experimental data, e.g. [1].

For full QCD with dynamical quarks one expects the deconfinement phase transition to be a crossover as quarks explicitly break the underlying center symmetry of the gauge group. The nature of the chiral phase transition primarily depends on the value of the current quark mass, which explicitly breaks chiral symmetry, as well as on the strength of the chiral anomaly [2]. While the confinement phase transition is driven by gluodynamics, the chiral phase transition is governed by strong quark interactions. It is a highly non-trivial observation that both lie remarkably close at least for small quark chemical potentials $[3,4]$. An understanding of this interrelation is subject of an ongoing debate.

Hence, the resolution of the QCD phase diagram requires both, the computation of the relevant observables, in particular the order parameters, and also some analytic understanding of the mechanisms involved, see e.g. [5]. Such a twofold task is best obtained within a combination of different methods that allow a direct access to the observables as much as to the mechanisms behind the observed phenomena. Functional continuum methods such as functional renormalisation group equations (FRG) and Dyson-Schwinger equations (DSE) are well-suited for the above task. They are indeed complementary to lattice simulations: most importantly they are amiable towards chiral fermions including the anomalous breaking of chiral symmetry and are straightforwardly applied to finite chemical potential. Ideally lattice simulations and func- tional approaches go hand in hand, and should be used to improve and to check respective results, also leading to better systematic error estimates. In combination, this should allow us to map out the phase diagram of QCD.

\section{Functional RG}

We shall present results on the order parameter for the chiral and the confinement-deconfinement phase transition obtained with functional methods, mostly with the FRG. Within a functional approach one usually computes correlations functions of quarks and gluons or that of composite operators such as hadronic degrees of freedom. These correlations functions are related by an infinite hierarchy of partial integro-differential equations which are solved within specific approximations to the full system at hand. Clearly a sound discussion of the approximations is of chief importance for the reliability of functional approaches. However, it is beyond the scope of the present overview, and for more details we defer the reader to the original works.

Most of the results presented here are obtained in Landau gauge QCD with the classical action

$$
\begin{aligned}
S_{\mathrm{QCD}}= & \frac{1}{4} \int_{x} F_{\mu \nu}^{a}{ }^{2}+\frac{1}{2 \xi} \int_{x}\left(\partial A^{a}\right)^{2}+\int_{x} \bar{C}^{a}(\partial D)^{a b} C^{b} \\
& +\int \bar{\psi}\left(i D D+i m_{\psi}+i \mu \gamma_{0}\right) \psi
\end{aligned}
$$

where $\xi \rightarrow 0$. An infrared regularisation is achieved with the introduction of momentum-dependent mass-terms for ghost, gluon and quark fields. We also introduce cutoff terms for effective low energy degrees of freedom such as mesons and baryons. This leads to a scaledependent effective action, $\Gamma_{k}[A, C, \bar{C}, \psi, \bar{\psi} ; \sigma, \vec{\pi}, \ldots]$, with infrared cut-off scale $k$. The $\sigma$ and $\vec{\pi}$ fields stand for mesonic composite operators. For $k \rightarrow 0$, we approach 
the fully interacting theory, whereas for $k \rightarrow \infty$ we are left with the asymptotically free high energy QCD. An infinitesimal change of the scale $k$ is described by Wetterich's flow equation, [6],

$$
\partial_{t} \Gamma_{k}[\phi]=\frac{1}{2} \operatorname{Tr}\left(\Gamma_{k}^{(2)}[\phi]+R_{k}\right)^{-1} \partial_{t} R_{k},
$$

where $t=\ln k / \Lambda$, and $\phi=(A, C, \bar{C}, \psi, \bar{\psi} ; \sigma, \vec{\pi}, \ldots)$. Finally, $\Gamma_{k}^{(2)}$ denotes the second derivative of $\Gamma_{k}$ w.r.t. the fields. The cut-off functions $R_{k}$ provide infrared cut-offs for all fields, including the composite fields, i.e. $\sigma$ and $\vec{\pi}$, for details see the reviews on gauge theories, $[7,8,9,10]$. We emphasise that (2) comprises a first principle QCD flow, the appearance of composite operators does not signal an effective field theory setting but rather a convenient parametrisation, see $[8,11]$. The flow (2) has a simple diagrammatic form depicted in Fig. 1, see [12]. The

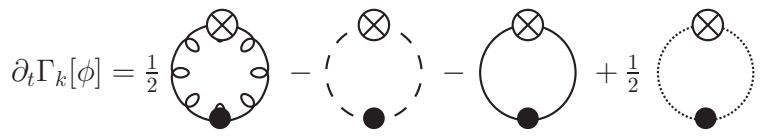

FIGURE 1. Functional flow for QCD: the lines denote full field dependent propagators. Crosses denote the cut-off insertion $\partial_{t} R$.

first and second loop generate gluon and ghost fluctuations respectively, the third loop generates fluctuations of the quarks, and the last loop stands for the loops of the mesonic $\sigma$ - and $\vec{\pi}$-fluctuations, and possible further hadronic degrees of freedom.

\section{Flows for Yang-Mills propagators in the Landau gauge}

The Landau gauge has very peculiar properties that facilitate the computation of correlations functions: first of all, the ghost-gluon vertex is protected from renormalisation. Second, we have infrared ghost dominance. Its weak form relevant for the present investigations simply entails that the gluon dressing function is vanishing in the infrared, $p^{2} / \Gamma^{(2)}(p \rightarrow 0) \rightarrow 0$, whereas the ghost dressing function does not, $p^{2} / \Gamma_{C}^{(2)}(p \rightarrow 0)>0$. For a detailed discussion of the global properties of Landau gauge Yang-Mills theory see [13, 14]. A related gauge with similar properties is Coulomb gauge, for a FRG study and further references see $[15,16]$.

The full set of flow equations for ghost and gluon propagators is derived from Fig. 1 with two derivatives w.r.t. the ghost fields and the gluons respectively. The propagator flows are coupled sets of integro-differential one loop equations for the propagators that also depend on vertex functions with up to four legs, see Fig. 2. This system of flow equations has been studied in $[13,17,18$, $19,20,21]$. In the following we use the numerical solutions of the FRG equations for the propagators in [13],

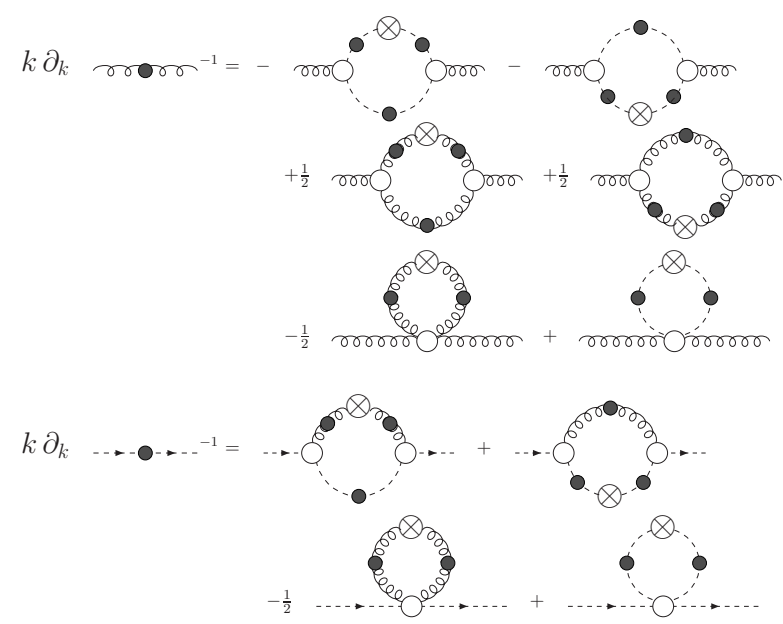

FIGURE 2. Functional RG equations for the gluon and ghost propagator. Filled circles denote dressed propagators and empty circles denote dressed vertex functions. Crosses denote the cut-off insertion $\partial_{t} R$.

the gluon propagator is displayed in Fig. 3. The vertices used for this solution are fully RG-dressed and depend on one (symmetric) momentum scale $k$. The four gluon vertex is partially $2 \mathrm{PI}$-resummed and hence includes also the sunset diagram with full propagators. If compared with similar DSE computations, see [13, 23, 24, 25, 26] and references therein, it is in particular the latter property which includes contributions that are contained in the two-loop diagrams in the DSEs. These diagrams are neglected in most approximations to DSEs (see however [27]). This fact is most probably responsible for the minor deviations of the DSE propagators from the lattice results, e.g. [22, 28, 29, 30], in the mid momentum regime around the peak of the gluon dressing function in Fig. 3, for more details see [13]. In spite of this both functional approaches provide results which are in quantitative agreement with the lattice propagators.

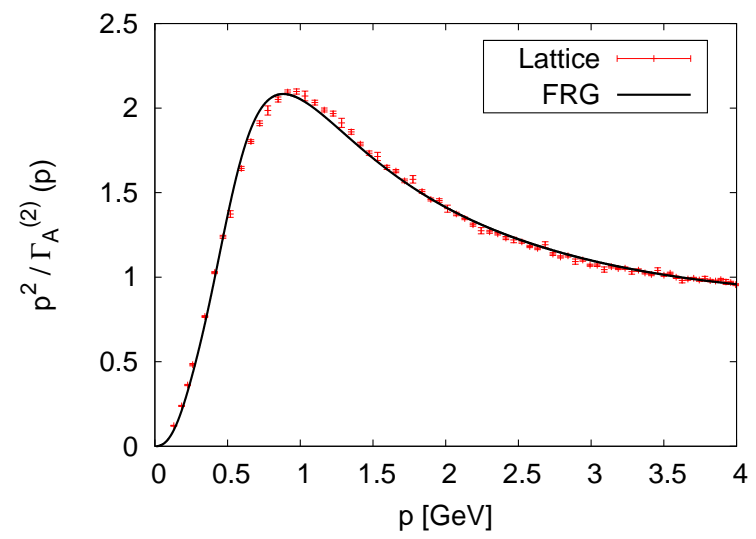

FIGURE 3. Gluon dressing function: FRG [13], lattice [22]. 
Quark confinement from Yang-Mills propagators

The Polyakov loop variable $L(\vec{x})$,

$$
L(\vec{x})=\frac{1}{N_{\mathrm{c}}} \operatorname{tr} \mathrm{P} \operatorname{expig} \int_{0}^{\beta} d t A_{0}(t, \vec{x}),
$$

in QCD with $N_{c}$ colors and infinitely heavy quarks is related to the operator that generates a static quark. The trace in (3) is evaluated in the fundamental representation, $\mathrm{P}$ stands for path ordering, and $\beta=1 / T$ is the inverse temperature. We can interpret the logarithm of the expectation value $\langle L\rangle$ as half of the free energy $F_{q \bar{q}}$ of a static quark-anti-quark pair at infinite distance. Moreover, $\langle L\rangle$ is an order parameter for the center symmetry of the gauge group, see e.g. [31].

It can be shown that $L\left[\left\langle A_{0}\right\rangle\right]$ also serves as an order parameter, [32, 33]: we have $L\left[\left\langle A_{0}\right\rangle\right] \geq\langle L\rangle$ with help of the Jensen inequality. We also have $L\left[\left\langle A_{0}\right\rangle\right] \equiv 0$ in the centersymmetric phase. Hence it vanishes exactly at $T_{\text {conf. }}$ Accordingly we have to simply find the solution to the equation of motion for $A_{0}: V_{\mathrm{YM}}^{\prime}\left[\left\langle A_{0}\right\rangle\right]=0$. The effective potential $V_{\mathrm{YM}}\left[A_{0}\right]$ is nothing but the effective action $\Gamma$ evaluated for constant fields $A_{0}, V_{\mathrm{YM}}\left[A_{0}\right]=\Gamma\left[A=A_{0}, C=\right.$ $0, \bar{C}=0]$. Its flow is governed by the ghost and gluon diagrams in Fig. 1, and hence can be computed solely from the ( $k$-dependent) ghost and gluon propagators [32]. In other words, $V_{\mathrm{YM}}$ being confining for low temperatures puts constraints on the behaviour of the ghost and gluon propagators in the deep infrared. Loosely speaking, confinement demands a minimal amount of infrared ghost dominance [32]. Interestingly, infrared stability in the background Landau gauge puts an upper bound on the amount of infrared ghost enhancement [34]. Together this puts rather tight constraints on the infrared asymptotics of the propagators which are satisfied by the actual numerical solutions [34]. Note, however, that the critical temperature is insensitive to the deep infrared [32, 35], see also [36, 37]. Moreover, confinement is not directly sensitive to the size of the coupling. These statements hold true in dynamical QCD and extends to other observables [12].

Inserting the propagators shown in Fig. 3 we get the effective potential shown in Fig. 4. The transition temperature is computed as $T_{c}=276 \pm 10 \mathrm{MeV}$ in quantitative agreement with the lattice result of $T_{c}=270 \mathrm{MeV}$, e.g. [38]. The order parameter, $L\left[\left\langle A_{0}\right\rangle\right]$ is obtained from the minimum of the potential in Fig. 4 and is shown in Fig. 5. If compared with lattice results for $\langle L\rangle$, e.g. [38], the Polyakov loop variable $L\left[\left\langle A_{0}\right\rangle\right]$ shows a steeper slope and hence a smaller transition region, see also [39], in accordance with the Jensen inequality discussed above. Note, however, that the results presented in Figs. 4 and 5 are obtained by neglecting the back-reaction of the $A_{0^{-}}$ fluctuations related to $V_{\mathrm{YM}}^{\prime \prime}\left[\left\langle A_{0}\right\rangle\right]$. These fluctuations reduce the slope of the Polyakov loop variable $L\left[\left\langle A_{0}\right\rangle\right]$. For

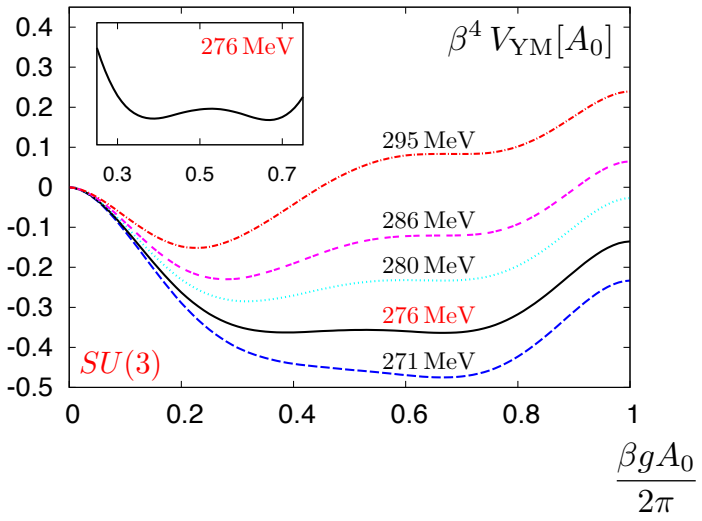

FIGURE 4. Polyakov loop effective potential [32].

a system at a second order phase transition they carry the universal properties of a system and drive the system into the symmetric phase. In turn, they are sub-leading for the critical temperature of a first order phase transition. This is one of the reasons for the good quantitative precision of the results for the critical temperature for $S U(3)$ and higher $S U(N)$ [35].

For $S U$ (2) Yang-Mills theory we have a second order phase transition (Ising universality class), and indeed we find $T_{\text {conf }} / \sqrt{\sigma}=.605$ instead of $T_{\text {conf }} / \sqrt{\sigma}=$ .709 [38], with string tension $\sigma$. An alternative computation of the order parameters in $S U(2)$ has been done in Polyakov gauge. There the flow is completely described by the $V_{\mathrm{YM}}^{\prime \prime}\left[A_{0}\right]$-fluctuations [33], and the critical temperature is computed as $T_{\text {conf }} / \sqrt{\sigma}=.69$. We also find the Ising class critical exponents. More recently, the Landau gauge computation has been extended to the $V_{\mathrm{YM}}^{\prime \prime}\left[A_{0}\right]$ fluctuations with $T_{\text {conf }} / \sqrt{\sigma}=.705$ [40]. A comparison of the temperature-dependence of the order parameter in the Polyakov gauge and the Landau gauge gives a remarkable agreement for all temperatures and provides strong support for the gauge independence of our results [33]. This concludes the investigation of Yang-Mills theory.

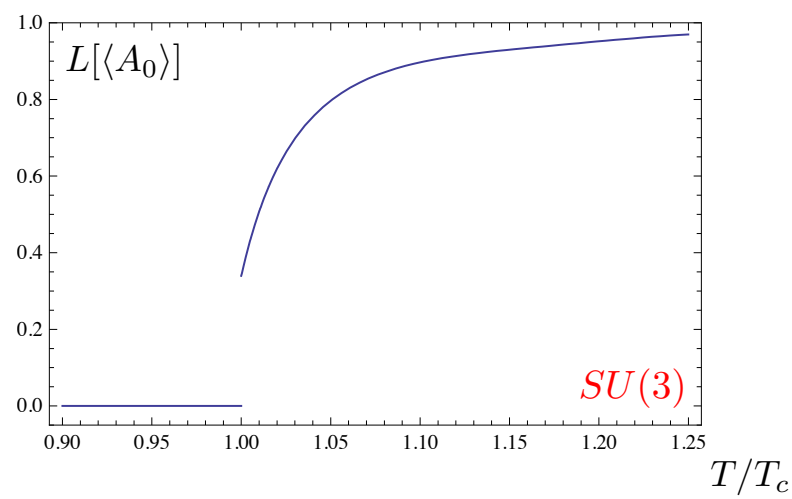

FIGURE 5. Polyakov loop [32]. 


\section{$Q C D$, chiral symmetry breaking and dynamical hadronisation}

The matter fluctuations due to dynamical quarks are encoded in the last two loops in Fig. 1. The quarkgluon interaction gives rise to four-fermi terms upon integration of a momentum shell with the flow. They originate from box diagrams $\propto \alpha_{s}^{2}$, leading to

$$
\int_{x} \lambda_{\psi, k}\left(\alpha_{s}\right)\left[(\bar{\psi} \psi)^{2}+\left(i \bar{\psi} \gamma_{5} \vec{\tau} \psi\right)^{2}\right],
$$

where $\tau=\left(\sigma_{1}, \sigma_{2}, \sigma_{3}\right)$. Here we have restricted ourselves to the two-flavour case, $N_{f}=2$. Further momentum shell integration then also give contributions to the four-fermi terms related to (4) as the flow $\partial_{t} \lambda_{\psi, k}=\partial_{t} \lambda_{\psi, k}\left(\lambda_{\psi}, \alpha_{s}\right)$. Note that for $\alpha_{s}=0$ this resembles a NJL-type flow. The four-fermi term in (4) can be conveniently rewritten with a Hubbard-Stratonovich transformation as

$$
\frac{1}{2} \int_{x} m_{\sigma, k}^{2}\left(\sigma^{2}+\vec{\pi}^{2}\right)+\int_{x} h_{k}\left[(\bar{\psi} \psi) \sigma+\left(i \bar{\psi} \gamma_{5} \vec{\tau} \psi\right) \vec{\pi}\right],
$$

with $m_{\sigma, k}^{2}=h_{k}^{2} /\left(2 \lambda_{\psi, k}\right)$ and using the equations of motion for $\sigma$ and $\vec{\pi}$. Further momentum shell integrations generate kinetic terms for the mesonic degrees of freedom $\sigma$ and $\vec{\pi}$ and further interaction terms, in particular an effective potential $V_{\text {eff, } \mathrm{k}}\left[\sigma^{2}+\vec{\pi}^{2}\right]$ which includes the mesonic mass in (5). Additionally the four-fermi interaction (4) is re-generated from the quark-gluon interaction. This already leads to an effective action $\Gamma_{k}[\phi]$ with $\phi=(A, C, \bar{C}, \psi, \bar{\psi} ; \sigma, \vec{\pi})$, and suffices to describe the onset of chiral symmetry breaking, [12, 41, 42, 43, 44]. The above structure has been also advocated in [45] on the basis of the pure glue results in [33]. An important feature of the above bosonisation is that it is not subject to double-counting problems. With cut-off terms for the mesonic degrees we are led to the flow equation (2) depicted in Fig. 1.

In this setting chiral symmetry breaking is monitored by the expectation value of $\sigma$ : the Yukawa term in (5) can be absorbed into the Dirac term in (1) by

$$
i m_{\psi, k} \bar{\psi} \psi \rightarrow \bar{\psi}\left(i m_{\psi, k}+h_{k}\left(\sigma+i \gamma_{5} \vec{\tau} \pi\right)\right) \psi .
$$

Hence, for a non-vanishing expectation value of $\sigma$ this term serves as an additional mass term for the quarks. We concentrate on the Yukawa term and the effective mesonic potential at vanishing pion field, $\vec{\pi}=0$,

$$
\int_{x} V_{\mathrm{eff}, \mathrm{k}}\left[\sigma^{2}\right]+\int_{x} h_{k}(\bar{\psi} \psi) \sigma .
$$

In the symmetric phase the effective potential $V_{\text {eff, }}$ has its minimum at $\sigma=0$ and the fermionic term vanishes on the equation of motion. In the broken phase the minimum is at $\bar{\sigma} \neq 0, V_{\text {eff }}^{\prime}\left[\bar{\sigma}^{2}\right]=0$ and the fermionic term gives rise to a mass term with mass $\left|h_{k}\right| \bar{\sigma}$. Indeed, $f_{\pi} \simeq$ $\left|h_{k}\right| \bar{\sigma}$ is an order parameter for the chiral phase transition which happens at $m_{\sigma}^{2}=0$, that is $\lambda_{\psi} \rightarrow \infty$. Note that even within this simplified setting this already introduces momentum-dependent (non-local) four-fermi couplings. Via the coupling to the effective mesonic potential and the kinetic terms one also generates (non-local) higher order fermionic terms.

The above approach is systematically improved by dynamical hadronisation or rebosonisation $[8,11,41$, 46]: the re-generated four-fermi interaction can be reabsorbed in the Yukawa-interaction in (5). This dynamically re-adjusts the expansion of the effective action in the scale-dependent relevant degrees of freedom and guarantees or at least improves the locality of the expansion in relevant degrees of freedom. In this way the system evolves dynamically from the high temperature/large cut-off scale quark-gluon phase to the low temperature/small cut-off scale hadronic phase, see in particular [44].

With or without dynamical hadronisation it is a particular strength of the present approach that it allows a direct access to the physics mechanisms. The rôle of the gauge coupling for chiral symmetry breaking is already easily displayed in the setting without (full) dynamical hadronisation. In the perturbative regime of QCD the four-fermi coupling can be safely put to zero. The aforementioned box diagrams then generate and successively increase the strength of the four-fermi coupling. If it exceeds a critical strength, spontaneous chiral symmetry breaking is induced similarly to the NJL model. In the present QCD approach, this is triggered by the increase of the gauge coupling $\alpha_{s}$ : for $\alpha_{s}<\alpha_{s, \text { crit }}$ spontaneous chiral symmetry breaking is triggered [12, 41, 42, 43]. In turn, if $\alpha_{s}$ does not exceed the critical coupling $\alpha_{s}>$ $\alpha_{s, \text { crit }}$, the four-fermi coupling never grows big and runs into the Gaußian infrared fixed point $\lambda_{\psi}=0$ with chiral symmetry. We conclude that chiral symmetry breaking is primarily driven by the strength of the gauge coupling in contradistinction to the deconfinement transition.

\section{Results: phase structure at vanishing density}

The approach described in the preceding sections is now put to work in two flavour QCD in the chiral limit and at vanishing density. We map out the confinementdeconfinement phase transition with the Polyakov loop order parameter $L\left[\left\langle A_{0}\right\rangle\right]$, and the pion decay constant $f_{\pi} \simeq\langle\sigma\rangle$. The confinement-deconfinement phase transition is also accessible by so-called dual order parameters which are derived from quark correlation functions $[36,47,48,49,50]$ with non-trivial temporal boundary conditions for the quark fields in the chosen correlator. In [12] it has been shown that such order parameters can be 


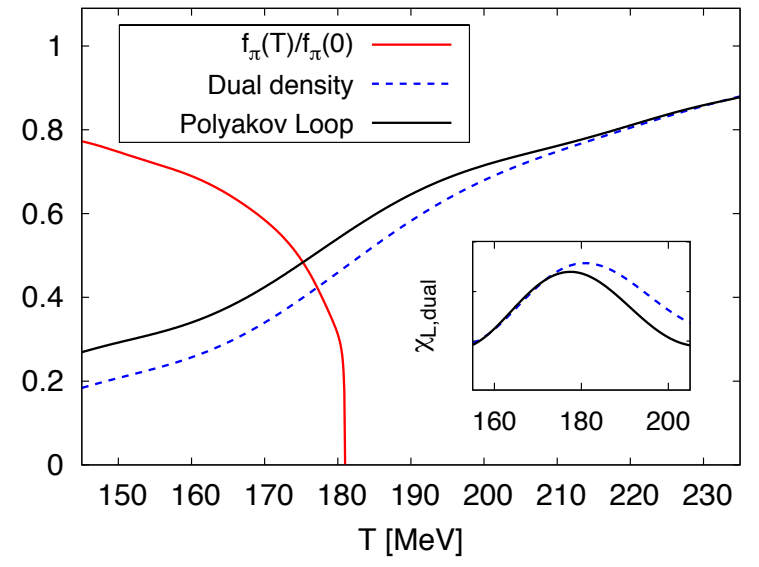

FIGURE 6. Pion decay constant, dual pressure difference and Polyakov loop as functions of temperature, $\chi_{L}=\partial_{T} L$, $\chi_{\text {dual }}=\partial_{T} \tilde{p}[12]$.

also derived from $\mathrm{QCD}_{\theta}$ at imaginary chemical potential $\mu=2 \pi i \theta / \beta$, for more details see also [51] in these proceedings. Here we only remark that imaginary chemical potential can be recast as a non-trivial boundary condition for the quarks,

$$
\int \bar{\psi}_{\theta}\left(i D D+i m_{\psi}\right) \psi_{\theta}, \quad \psi_{\theta}(t+\beta, \vec{x})=-e^{2 \pi i \theta} \psi(t, \vec{x}) .
$$

It can be shown that the first Fourier moment in $\theta$ of any observable is sensitive to center symmetry $[12,51]$. Hence, if it does not vanish identically, it is an order parameter for the confinement-deconfinement phase transition. This allows to define the so-called dual pressure (or density) as an order parameter for the confinementdeconfinement phase transition (in pure Yang-Mills),

$$
\tilde{p}=\int_{0}^{\theta} d \theta e^{-2 \pi i \theta} \Gamma_{\theta}\left[\left\langle A_{0}\right\rangle_{\theta=0},\langle\sigma\rangle_{\theta=0}\right]
$$

Here, $\Gamma_{\theta}$ is the effective action of $\mathrm{QCD}_{\theta}$, and $\left\langle A_{0}\right\rangle_{\theta=0}$ and $\langle\sigma\rangle_{\theta=0}$ are the solutions of the equations of motion in the physical theory at $\theta=0$. The advantage of the order parameter (9) is, that its flow $\partial_{t} \tilde{p}$ is directly related to the flow of the effective action, (2). Clearly, this is the object least sensitive to a given approximation of $\Gamma_{k}$.

The results for the order parameters are displayed in Fig. 6 and suggest a relation between the chiral phase transition and the broad confinement-deconfinement cross-over with a width of approximately $20 \mathrm{MeV}$, both happen at about $180 \mathrm{MeV}$. The agreement between the cross-over temperature derived from the Polyakov loop and the dual pressure is remarkable as is the apparent close relation of their values for all temperatures. The latter has been studied in more detail in $[12,51]$ and we defer the reader to this work. Beside its formal relation

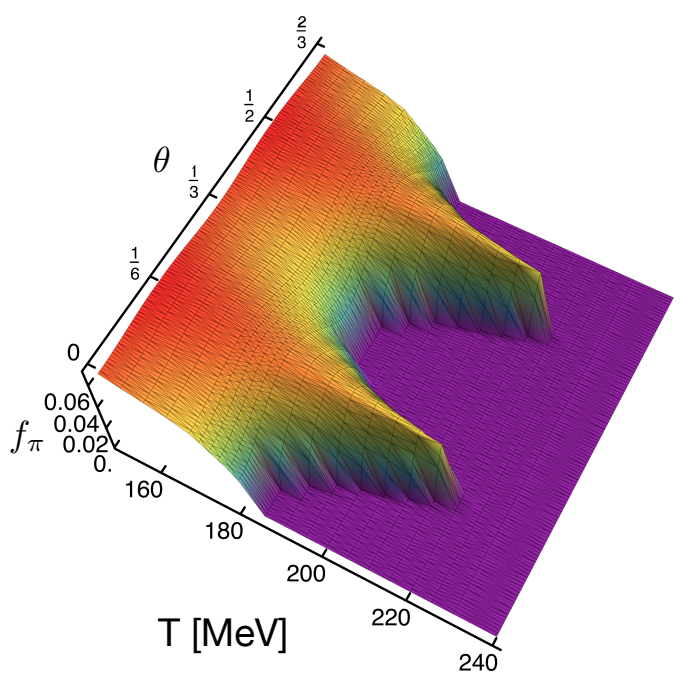

FIGURE 7. Pion decay constant as a function of imaginary chemical potential and temperature [12].

it constitutes a non-trivial consistency check of the approximation used as the Polyakov loop is dominated by gluonic fluctuations whereas the dual pressure is dominated by matter fluctuations. Finally we would like to remark that qualitatively our findings compare well with the lattice findings for $2+1$ flavours, see e.g. $[52,53,54,55,56]$.

\section{Results: phase structure at finite density}

A first step towards non-vanishing density is the inclusion of an imaginary chemical potential. It allows us to compute the dual order parameters such as the dual pressure (9). We also can collect indirect information about real chemical potential by continuation. In specific cases this may allow to fix the phase structure at real chemical potential [57].

The pion decay constant is displayed in Fig. 7. Together with the Polyakov loop it leads to the phase structure displayed in Fig. 8. We conclude that the close relation between the chiral phase transition and the confinement-deconfinement cross-over persists at imaginary chemical potential. This is in agreement with related lattice computations at relatively heavy quark masses, e.g. $[4,58]$. In contradistinction, in model computations this is only seen within an adjustment of the coupling of eight-fermi interaction [59]. In the present QCD approach these terms with their QCD-induced coupling are generated by the flow, no adjustment is required. This pattern allows us to access the interesting question of the nature of the Roberge-Weiss endpoint. This is important for the extension of the computations at imaginary chemical potential to real chemical potential, [57,60], and is currently under investigation in the present approach. 


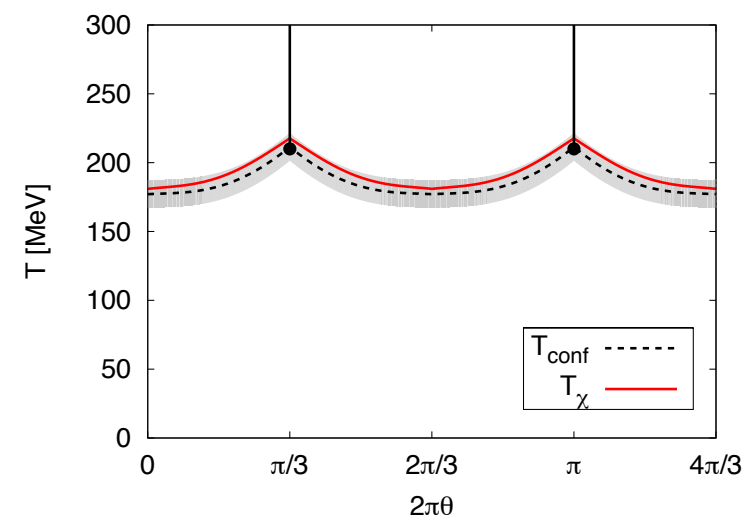

FIGURE 8. QCD phase diagram at imaginary chemical potential. The grey band represents the width of $\chi_{L}$. Black dots indicate the endpoints of the Polyakov loop RW transitions [12].

Finally we consider real chemical potential. As has been stressed before, functional approaches do not suffer from the sign problem that is so virulent within lattice simulations at real chemical potential. A FRG study of the chiral phase boundary in one-flavour QCD has been put forward in [44]. The results include in particular the curvature of the chiral phase boundary which is consistent with lattice results for small chemical potential.

First computations within two-flavour QCD at real chemical potential have also been performed. Most notably the two critical temperatures stay close to each other; indeed, the confinement-deconfinement temperature tends to get smaller in comparison to the chiral one. This is not seen in most computations with Polyakov loop-extended models and is related to the missing backcoupling of the matter sector into the glue sector in these models. To see this more clearly we use the fact that the above-mentioned models, e.g. [61, 62, 63, 59] and their non-local versions, e.g. [64], can be interpreted as specific approximations of the fully dynamical QCD flow in [12], for more details on this relation see also [65]. We first remark that the matter sector including fluctuations is given by the last two matter diagrams in Fig. 1. Switching off the glue contributions to the matter propagators leads to general quark-meson models which have been studied intensively beyond mean field with the FRG, for reviews see e.g. [66, 67, 68].

In turn, the glue part of QCD is encoded in the first two, pure glue, diagrams. Switching off the matter contributions to the glue dynamics reduces the glue part to Yang-Mills theory, and the Polyakov loop potential reduces to that of Yang-Mills theory. In the Polyakov loopextended models, the coupling of these two, now decoupled, sectors is reintroduced via the $A_{0^{-}}$(or Polyakov loop $L$ ) and $\sigma$-background dependence of the quark loop. Note that such an approximation of the QCD flow in

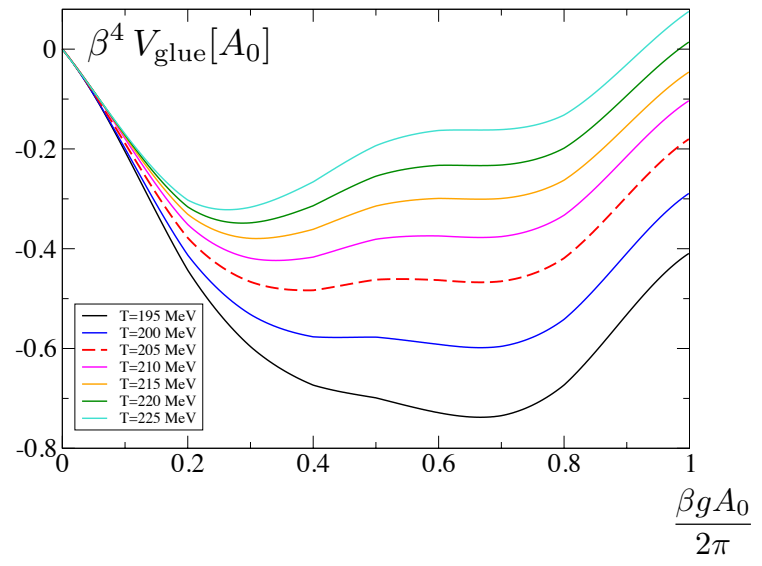

FIGURE 9. Pure glue part of the Polyakov loop potential in two-flavour QCD.

Fig. 1 still involves a fluctuating matter sector. This describes these models beyond mean field as has been studied in $[65,69,70]$.

The above connection of the Polyakov loop-extended models to the present QCD approach can be used to qualitatively improve these models: the full QCD effective potential (or grand potential) $V_{\mathrm{QCD}}$ is obtained by evaluating the integrated flow in Fig. 1 for constant $A_{0}$ - and $\sigma$-fields [12],

$$
V_{\mathrm{QCD}}=V_{\text {glue }}\left[A_{0}, \sigma\right]+V_{\text {quark }}\left[A_{0}, \sigma\right]+V_{\text {meson }}\left[A_{0}, \sigma\right] .
$$

The glue potential $V_{\text {glue }}$ is computed from the ghost and gluon loop in Fig. 1, $V_{\text {quark }}$ is computed from the quark loop, and $V_{\text {meson }}$ is computed from the mesonic loop. $V_{\text {quark }}+V_{\text {meson }}$ are the direct matter contributions to the full Polyakov loop potential. We emphasise that the propagators in this computation are that of fully-coupled QCD with dynamical quarks and mesons [12].

We have already remarked that $V_{\text {glue }}$ is approximated by the Yang-Mills potential $V_{\mathrm{YM}}$ in the Polyakov loopextended models. With $V_{\text {glue }}$ from the QCD flows in [12] this approximation can be resolved which will be detailed elsewhere. Here we discuss how to properly adjust the parameters in the Yang-Mills potential shown in Fig. 4 by comparing it to the full glue potential. For this purpose we evaluate $V_{\text {glue }}$ on the solution $\sigma_{0}$ of the equations of motion, $V_{\text {glue }}\left[A_{0}\right]=V_{\text {glue }}\left[A_{0}, \sigma_{0}\right]$ as shown in Fig. 9. It turns out that the related Polyakov loop compares well with the Yang-Mills one in Fig. 5 if both are plotted as functions of $T / T_{c}$. Indeed, the form of the pure glue potential is qualitatively unchanged in comparison to the Yang-Mills potential, and still shows a first order phase transition. The latter has to be taken with a grain of salt as we have dropped the center-breaking, though sub-leading, $V_{\mathrm{Q} C D}^{\prime \prime}$-terms in the gluon propagator. In any case, the transition temperature is significantly reduced, 


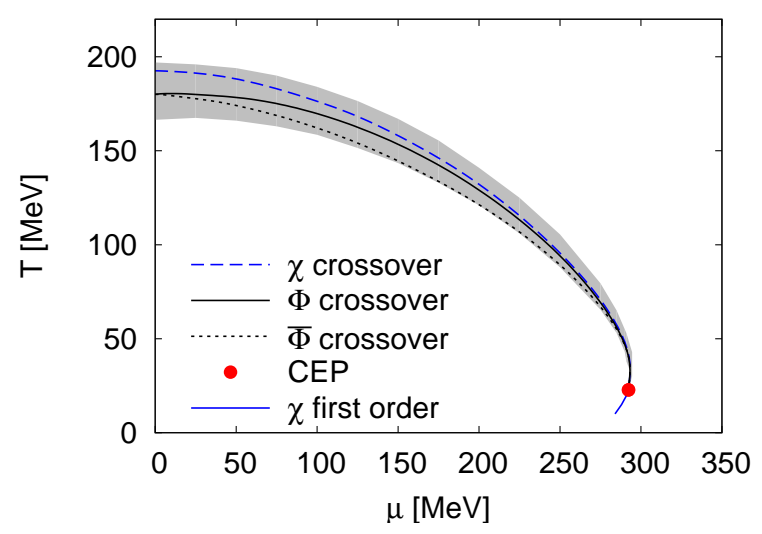

FIGURE 10. Chiral and deconfinement phase diagram in the PQM model. The (grey) band corresponds to the width of $d \Phi / d T$ at $80 \%$ of its peak height [65].

from $T_{\mathrm{YM}}=276 \mathrm{MeV}$ to $T_{\text {glue }} \approx 205 \mathrm{MeV}$. We conclude that the full glue potential is modeled well by the YangMills potential with a reduced critical temperature. We also remark that the above procedure allows in general to adjust the sensitive parameters of the Polyakov loopextended models with QCD-input. Most importantly, this allows for a systematic improvement of these models towards full QCD.

The above analysis confirms quantitatively the phenomenological HTL/HDL estimate in [61]. Using the latter also for larger chemical potential as well as utilising FRG flows for the matter fluctuations in the presence of a Polyakov loop we are led to the phase diagram displayed in Fig. 10, see [65]. At large densities or large chemical potential the present approximation without baryons certainly is not trustworthy, in particular in the hadronic phase. The important inclusion of baryons shall be put forward with dynamical hadronisation. Nonetheless the combined results from both the model computations and the QCD flows constrains the position of the critical point; a conservative estimate leads to $\mu / T>2 / 3$.

\section{Conclusions}

In summary we have put forward a quantitative functional approach to the phase diagram of QCD at finite temperature and density. So far fresh insight has been gained in Yang-Mills theory, in one-flavour and in twofavour QCD at finite temperature and density. The results compare well to lattice computations without the fixing of additional parameters. However, the approach also provides results beyond the applicability range of lattice simulations, in particular at large density. Currently it is extended to $2+1$ flavours as well as to baryons.
Acknowledgments. I thank the organisers for hosting such a stimulating conference. I thank J. Braun, A. Eichhorn, C. S. Fischer, L. Fister, H. Gies, L. M. Haas, T. K. Herbst, A. Maas, F. Marhauser, B.-J. Schaefer, F. Spallek and L. von Smekal for discussions and a pleasant collaboration on the work presented here. This work is supported by Helmholtz Alliance HA216/ EMMI.

\section{REFERENCES}

1. P. Braun-Munzinger, K. Redlich, and J. Stachel (2003), nucl-th/0304013.

2. R. D. Pisarski, and F. Wilczek, Phys. Rev. D29, 338-341 (1984).

3. Y. Aoki, Z. Fodor, S. Katz, and K. Szabo, Phys.Lett. B643, 46-54 (2006), hep-lat / 0609068.

4. P. de Forcrand, and O. Philipsen, Nucl. Phys. B642, 290-306 (2002), hep-lat / 0205016.

5. R. Alkofer, et al. (2010), 1012.3192.

6. C. Wetterich, Phys. Lett. B301, 90-94 (1993).

7. D. F. Litim, and J. M. Pawlowski (1998), hep-th/9901063.

8. J. M. Pawlowski, Annals Phys. 322, 2831-2915 (2007), hep-th/0512261.

9. H. Gies (2006), hep-ph/ 0611146.

10. Y. Igarashi, K. Itoh, and H. Sonoda, Prog. Theor. Phys. Suppl. 181, 1-166 (2010), 0909.0327.

11. H. Gies, and C. Wetterich, Phys. Rev. D65, 065001 (2002), hep-th/0107221.

12. J. Braun, L. M. Haas, F. Marhauser, and J. M. Pawlowski, Phys. Rev. Lett. In Press (2011), 0908.0008.

13. C. S. Fischer, A. Maas, and J. M. Pawlowski, Annals Phys. 324, 2408-2437 (2009), 0810.1987.

14. L. von Smekal (2008), 0812.0654.

15. M. Leder, J. M. Pawlowski, H. Reinhardt, and A. Weber (2010), 1006.5710.

16. H. Reinhardt, M. Leder, J. M. Pawlowski, and A. Weber (2010), 1011.3462.

17. U. Ellwanger, M. Hirsch, and A. Weber, Z. Phys. C69, 687-698 (1996), hep-th/9506019.

18. U. Ellwanger, M. Hirsch, and A. Weber, Eur. Phys. J. C1, 563-578 (1998), hep-ph/9606468.

19. B. Bergerhoff, and C. Wetterich, Phys. Rev. D57, 1591-1604 (1998), hep-ph/9708425.

20. J. M. Pawlowski, D. F. Litim, S. Nedelko, and L. von Smekal, Phys. Rev. Lett. 93, 152002 (2004), hep-th/0312324.

21. C. S. Fischer, and H. Gies, JHEP 10, 048 (2004), hep-ph/0408089.

22. A. Sternbeck, E. M. Ilgenfritz, M. Muller-Preussker, A. Schiller, and I. L. Bogolubsky, PoS LAT2006, 076 (2006), hep-lat/0610053.

23. R. Alkofer, and L. von Smekal, Phys.Rept. 353, 281 (2001), hep-ph/0007355.

24. C. S. Fischer, J. Phys. G32, R253-R291 (2006), hep-ph/0605173.

25. D. Binosi, and J. Papavassiliou, Phys. Rept. 479, 1-152 (2009), 0909.2536.

26. R. Alkofer, C. S. Fischer, M. Q. Huber, F. J. LlanesEstrada, and K. Schwenzer, PoS CONFINEMENT8, 019 (2008), 0812.2896. 
27. J. C. Bloch, Few Body Syst. 33, 111-152 (2003), hep-ph/0303125.

28. O. Oliveira, and P. Silva, Eur.Phys.J. C62, 525-534 (2009), 0705.0964.

29. A. Cucchieri, and T. Mendes, PoS LAT2007, 297 (2007), 0710.0412.

30. I. Bogolubsky, E. Ilgenfritz, M. Muller-Preussker, and A. Sternbeck, PoS LAT2007, 290 (2007), 0710.1968.

31. J. Greensite, Prog.Part.Nucl.Phys. 51, 1 (2003), hep-lat/0301023.

32. J. Braun, H. Gies, and J. M. Pawlowski, Phys. Lett. B684, 262-267 (2010), 0708.2413.

33. F. Marhauser, and J. M. Pawlowski (2008), 0812 . 1144.

34. A. Eichhorn, H. Gies, and J. M. Pawlowski (2010), 1010.2153.

35. J. Braun, A. Eichhorn, H. Gies, and J. M. Pawlowski, Eur. Phys. J. C70, 689-702 (2010), 1007.2619.

36. C. S. Fischer, Phys. Rev. Lett. 103, 052003 (2009), 0904.2700.

37. C. S. Fischer, A. Maas, and J. A. Muller, Eur. Phys. J. C68, 165-181 (2010), 1003.1960.

38. B. Lucini, M. Teper, and U. Wenger, JHEP 01, 061 (2004), hep-lat/0307017.

39. A. Dumitru, Y. Guo, Y. Hidaka, C. P. K. Altes, and R. D. Pisarski (2010), 1011 . 3820.

40. J. Braun, H. Gies, J. M. Pawlowski, and F. Spallek (2010), in preparation.

41. H. Gies, and C. Wetterich, Phys. Rev. D69, 025001 (2004), hep-th/0209183.

42. H. Gies, and J. Jaeckel, Eur. Phys. J. C46, 433-438 (2006), hep-ph/0507171.

43. J. Braun, and H. Gies, JHEP 06, 024 (2006), hep-ph/0602226.

44. J. Braun, Eur. Phys. J. C64, 459-482 (2009), 0810.1727.

45. K.-I. Kondo, Phys. Rev. D82, 065024 (2010), 1005.0314.

46. S. Floerchinger, and C. Wetterich, Phys. Lett. B680, 371-376 (2009), 0905 . 0915.

47. C. Gattringer, Phys. Rev. Lett. 97, 032003 (2006), hep-lat/0605018.

48. F. Synatschke, A. Wipf, and C. Wozar, Phys. Rev. D75, 114003 (2007), hep-lat/ 0703018.

49. E. Bilgici, F. Bruckmann, C. Gattringer, and C. Hagen, Phys. Rev. D77, 094007 (2008), 0801.4051.

50. B. Zhang, F. Bruckmann, C. Gattringer, Z. Fodor, and K. K. Szabo (2010), 1012.2314.

51. L. M. Haas, J. Braun, and J. M. Pawlowski (2010), 1012.4735.

52. Y. Aoki, S. Borsanyi, S. Durr, Z. Fodor, S. D. Katz, et al., JHEP 0906, 088 (2009), 0903.4155.

53. M. Cheng, S. Ejiri, P. Hegde, F. Karsch, O. Kaczmarek, et al., Phys.Rev. D81, 054504 (2010), 0911.2215.

54. S. Borsanyi, Z. Fodor, C. Hoelbling, S. D. Katz, S. Krieg, et al. (2010), 1011.4230.

55. A. Bazavov, and P. Petreczky (2010), 1012. 1257.

56. K. Kanaya (2010), 1012.4235.

57. P. de Forcrand, and O. Philipsen, Phys. Rev. Lett. 105, 152001 (2010), 1004.3144.

58. M. D'Elia, and M.-P. Lombardo, Phys. Rev. D67, 014505 (2003), hep-lat/0209146.

59. Y. Sakai, K. Kashiwa, H. Kouno, and M. Yahiro, Phys.Rev. D77, 051901 (2008), 0801.0034.
60. M. D'Elia, and F. Sanfilippo, Phys. Rev. D80, 111501 (2009), 0909.0254.

61. B.-J. Schaefer, J. M. Pawlowski, and J. Wambach, Phys. Rev. D76, 074023 (2007), 0704 . 3234.

62. K. Fukushima, Phys.Lett. B591, 277-284 (2004), hep-ph/0310121.

63. C. Ratti, M. A. Thaler, and W. Weise, Phys.Rev. D73, 014019 (2006), hep-ph / 0506234.

64. T. Hell, S. Roessner, M. Cristoforetti, and W. Weise, Phys.Rev. D79, 014022 (2009), 0810.1099.

65. T. K. Herbst, J. M. Pawlowski, and B.-J. Schaefer, Physics Letters $B$ In Press (2010), 1008.0081.

66. J. Berges, N. Tetradis, and C. Wetterich, Phys.Rept. 363, 223-386 (2002), hep-ph/ 0005122.

67. B.-J. Schaefer, and J. Wambach, Nucl. Phys. A757, 479-492 (2005), nucl-th/ 0403039.

68. B.-J. Schaefer, and J. Wambach, Phys. Part. Nucl. 39, 1025-1032 (2008), hep-ph / 0611191.

69. V. Skokov, B. Stokic, B. Friman, and K. Redlich, Phys.Rev. C82, 015206 (2010), 1004.2665.

70. V. Skokov, B. Friman, and K. Redlich (2010), 1008.4570 . 